\title{
Fratura exposta supracondiliana umeral e lesão vásculo-nervosa em paciente pediátrico
}

Supracondilian exposure fracture, uva and vascular-nervous injury in pediatric patient

Fractura de exposición supracondilia, uva y lesión nerviosa vascular en paciente pediátrico

Amanda Oliva SPAZIANI ${ }^{1}$

Guilherme Ruiz POLATTO ${ }^{1}$

Gustavo Faleiro BARBOSA ${ }^{1}$

João Gabriel Goulart ZANON ${ }^{1}$

Raulcilaine Érica dos SANTOS ${ }^{1}$

Talita Costa BARBOSA ${ }^{1}$

Natasha Ohana Marinho ROSA ${ }^{1}$

Lindemberg BARBOSA JÚNIOR ${ }^{2}$

Raissa Silva FROTA ${ }^{3}$

Flávio Henrique Nuevo Benez dos SANTOS ${ }^{4}$

'Universidade Brasil, Medicina. Fernandópolis - SP, Brasil

${ }^{2}$ Universidade Federal de Mato Grosso do Sul, Medicina. Três Lagoas - MS, Brasil

${ }^{3}$ Universidade de Rio Verde, Medicina. Goianésia - GO, Brasil

${ }^{4}$ Universidade Brasil, Médico, Esp. Ortopedia e Traumatologia, Esp. Medicina Física e Reabilitação, Me. Ciências da Saúde, MBA Gestão em Saúde. Fernandópolis-SP, Brasil

\section{Resumo}

Introdução: Fraturas e luxações na região do cotovelo são frequentes, principalmente na faixa etária pediátrica, correspondendo a 17\% das fraturas deste grupo. Objetivo: O presente trabalho objetiva explanar a fratura exposta supracondiliana umeral com lesão vásculonervosa em paciente pediátrico por meio de relato de caso e estudos de embasamento teórico. Metodologia: Trabalho realizado por meio de coleta de dados do prontuário médico e de artigos científicos. Sexo masculino, 7 anos, atendido em emergência após queda de cavalo e traumatismo em cotovelo esquerdo com exposição óssea em um ferimento maior do que dez centímetros. Relato de caso: Sendo assim, uma fratura exposta supracondiliana em cotovelo esquerdo - grau $3 \mathrm{C}$ - com lesão da artéria braquial e dos nervos radial e ulnar. Foi encaminhado para centro cirúrgico, onde foi realizada lavagem, desbridamento cirúrgico, redução e fixação da fratura. Foi realizada a revascularização da artéria braquial e neurorrafia dos nervos radial e ulnar. Fechamento por planos, curativo, tala e antibióticoterapia. Evoluiu com retorno da perfusão e pulsos radial e ulnar. Após três semanas, houve consolidação da fratura com pulsos preservados, mas com déficits nos nervosos. Após dois meses de reabilitação, já apresentava arco total do movimento do cotovelo, com seis, esboço dos nervos radial e ulnar e com um ano retorno de suas funções normais. Conclusão: Ainda são muitas as contradições em relação ao tipo de tratamento que deve ser instituído, justamente por ser uma região delicada e repleta de estruturas anatômicas que referem a um complexo sistema integrado por ossos, ligamentos, músculos, sistema vascular e neurológico que propiciam muitas vezes o processo iatrogênico durante a intervenção.

Descritores: Fraturas Ósseas; Fixação de Fratura; Cotovelo.

\section{Abstract}

Introduction: Fractures and dislocations in the elbow region are frequent, especially in the pediatric age group, corresponding to $17 \%$ of fractures in this group. Objective: The present work aims to explain the humeral supracondylar open fracture with vascular-nerve injury in a pediatric patient through case report and theoretical basis studies. Methodology: Work performed through data collection from medical records and scientific articles. Male, 7 years, treated in emergency after horse fall and left elbow trauma with bone exposure in an injury larger than 10 centimeters. Case report: Thus, an open supracondylar fracture in the left elbow - grade 3C - with brachial artery and radial and ulnar nerve injury. He was referred to the operating room, where he was washed, surgically debrided, reduced and fixed the fracture. Revascularization of the brachial artery and neurorraphy of the radial and ulnar nerves were performed. Flat closure, dressing, splint and antibiotic therapy. It evolved with return of perfusion and radial and ulnar pulses. After three weeks, the fracture was consolidated with preserved pulses, but with nerve deficits. After two months of rehabilitation, he already had a full arc of elbow movement, with six, outline of the radial and ulnar nerves and one year return to their normal functions. Conclusion: There are still many contradictions regarding the type of treatment that should be instituted, precisely because it is a delicate region full of anatomical structures that refer to a complex system composed of bones, ligaments, muscles, vascular and neurological system that provide many sometimes the iatrogenic process during the intervention.

Descriptors: Fractures, Bone; Fracture Fixation; Elbow.

\section{Resumen}

Introducción: las fracturas y dislocaciones de codo son frecuentes, especialmente en el grupo de edad pediátrica, y representan el $17 \%$ de las fracturas en este grupo. Objetivo: El presente trabajo tiene como objetivo explicar la fractura abierta supracondílea humeral con lesión del nervio vascular en un paciente pediátrico a través de un informe de caso y estudios de base teórica. Metodología: Trabajo realizado a través de la recolección de datos de registros médicos y artículos científicos. Varón de 7 años de edad, tratado en emergencia después de una caída del caballo y un traumatismo en el codo izquierdo con exposición ósea en una lesión de más de 10 centímetros. Caso clínico: por lo tanto, una fractura supracondílea abierta en el codo izquierdo - grado 3C - con arteria braquial y lesión del nervio radial y cubital. Fue derivado al quirófano, donde fue sometido a lavado, desbridamiento quirúrgico, reducción y fijación de la fractura. Se realizó revascularización de la arteria braquial y neurorrafia de los nervios radial y cubital. Cierre plano, vendaje, férula y tratamiento antibiótico. Evolucionó con el retorno de la perfusión y los pulsos radial y cubital. Después de tres semanas, la fractura se consolidó con pulsos conservados, pero con déficit nervioso. Después de dos meses de rehabilitación, ya tenía un arco completo de movimiento del codo, con seis, contorno de los nervios radial y cubital y un año de regreso a sus funciones normales. Conclusión: Todavía hay muchas contradicciones con respecto al tipo de tratamiento que debe instituirse, precisamente porque es una región delicada llena de estructuras anatómicas que se refieren a un sistema complejo compuesto de huesos, ligamentos, músculos, sistema vascular y neurológico que proporcionan muchos a veces el proceso iatrogénico durante la intervención.

Descriptores: Fracturas Óseas; Fijación de Fractura; Codo.

INTRODUÇÃO

Do ponto de vista médico, a luxação pode ser entendida como a "perda do contato articular". Assim definida, portanto, é a afastamento de dois ossos que habitualmente estão em íntimo e contínuo contato por meio de uma área lisa e deslizante, denominada de cartilagem $^{1,2}$. Eventualmente pode acontecer um trauma no braço por uma queda com a mão espalmada ou mesmo um choque no próprio cotovelo, e, nessa situação há a presença de 
dor, hematoma ou insuficiência de movimentos articulares, na maioria das vezes há uma fratura no cotovelo ${ }^{1,2}$.

Fraturas e luxações na região do cotovelo são frequentes, principalmente nas crianças que ainda estão na faixa etária considerada pediátrica, em termos percentuais as fraturas desse grupo são representadas por $17 \%$ do total de fraturas. Contudo, as fraturas supracondilianas umerais correspondem a cerca de 60 a $75 \%$ das fraturas pediátricas em membros superiores ${ }^{1,2}$.

As fraturas supracondilares recebem também a denominação de fraturas de Malgaigne. Essa lesão é encontrada, normalmente na parte superior da fossa olecrariana e tem por característica a desagregação da diáfise umeral dos côndilos do úmero distal. As linhas de fratura podem se estender para a região distal envolvendo a superfície articular ${ }^{3}$. A estrutura da lesão é responsável pela subdivisão dos tipos de fraturas supracondilares, permitindo estar presente após flexão ou após extensão do membro.

As fraturas que acontecem com maior frequência são em extensão, as supracondilares, essas fraturas são normalmente originadas por queda sobre a mão associada ou não a forças de abdução e adução. Em imagens radiográficas pode observar-se uma linha de fratura que passa do sentido ântero-distal para póstero-proximal ${ }^{4}$. Entretanto, as que têm menor incidência são as fraturas supracondilares do tipo flexão, sua origem se dá por meio de um trauma com força posterior sobre um cotovelo fletido gerando uma fratura oblíqua de ântero-proximal a pósterodistal. Outra característica relevante do trauma em flexão é a fratura exposta, resultado de um fragmento proximal deslocado que perfura 0 tríceps e a pele ${ }^{4}$. A classificação mais utilizada atualmente tem por base esse tipo de fratura em adultos, porém é possível ser utilizada para a faixa pediátrica, e é conhecida como classificação de Gartland ${ }^{3}$. Tendo três tipos de classificação, assim denominadas: tipo I - sem desvio; tipo II - com desvio e com a cortical posterior íntegra; e tipo III - com desvio e sem contato entre as corticais dos fragmentos ósseos ${ }^{5}$.

O cotovelo apresenta especificidades anatômicas, o terço distal do úmero oferece uma área óssea delgada entre as fossas do olécrano e coronóide, cercada pelas colunas medial e lateral, que são mais espessas. A fratura acontece quando há a queda do paciente com o cotovelo em hiperextensão, impactando o olécrano contra a fossa olecraneana, criando uma alavanca, cujo fulcro do movimento está localizado exatamente na região supracondiliana do úmero. Os pacientes pediátricos possuem maior lassidão ligamentar, que possibilita a hiperextensão do cotovelo, fato esse que facilita a ocorrência da fratura, por isso o pico de incidência se dá entre os 4 e 7 anos de idade ${ }^{2}$.

Aproximadamente $95 \%$ dos casos é referente ao mecanismo de trauma por hiperextensão. $O$ traço de fratura habitualmente é transverso e desloca-se no sentido pósterosuperior para ântero-inferior. É possível que o fragmento distal desvie em $75 \%$ dos casos, para posterior ou para posterior em varo (pósteromedial), ou em valgo (póstero-lateral). No caso da queda ocorrer com o cotovelo em semiflexão (menos de $5 \%$ dos casos), o fragmento desviase anteriormente, o traço de fratura é oblíquo, de ântero-superior para póstero-inferior, o que torna mais difícil sua redução, bem como a manutenção estável dos fragmentos ${ }^{2}$.

O diagnóstico passa pela anamnese e um rigoroso exame físico com o intuito de definir o triângulo utilizando-se a técnica do triângulo de Nélaton e a linha de Heuter que ficarão alteradas em caso de fratura ou luxação ${ }^{6}$. A sintomatologia caracteriza-se por dor alta, limitação funcional, edema, equimose e a depender da energia do trauma acontece a deformidade e lesão dos tecidos moles que envolvem o cotovelo. Complementarmente, o diagnóstico pode ser auxiliado prontamente por exames de imagem. A radiografia simples já é suficiente para definir o diagnóstico e realizar o planejamento do tratamento ${ }^{2}$. Não se pode desprezar a área vascular e neurológica do membro superior, pois existem estruturas anatômicas importantes que podem ser lesadas com o trauma, fato este que requer um exame minucioso e detalhado dessa área ${ }^{6}$.

Recomenda-se, geralmente, o tratamento para este tipo de lesão, a intervenção cirúrgica, porém não são descartados outros meios de tratamento e intervenção. As lesões em extensão minimamente deslocadas ou incompletas podem ser tratadas de forma não cirúrgica imobilizando com tala posterior a $90^{\circ}$ de flexão do cotovelo e antebraço na posição neutra, associado à reabilitação. Idosos com fraturas gravemente cominutivas cuja capacidade funcional é limitada também podem receber intervenção conservadora ${ }^{4}$. Já as fraturas em extensão abertas, deslocadas ou associadas com lesão vascular são tratadas com redução cirúrgica aberta e fixação com placas, uma em 
cada coluna, tanto paralelas quanto perpendiculares entre si proporcionando uma mobilização precoce.

Em pacientes idosos considera-se importante avaliar a possibilidade de artroplastia de substituição. Já as fraturas, provenientes de trauma em flexão, com deslocamento mínimo ou do tipo incompleta devem ser imobilizadas em relativa extensão, observado em todas as situações que o quadro clinico pode se agravar na medida em que gera deslocamento. As fraturas em flexão utilizam o mesmo processo cirúrgico do tipo extensão ${ }^{4}$. $O$ acesso cirúrgico ao cotovelo oferece dificuldades pela sua anatomia peculiar. Os elementos ósteoligamentares têm estreita relação com as estruturas nervosas e circulatórias. Em muitas ocasiões tem-se a necessidade de acessar mais de uma região do cotovelo realizando duas vias de acesso: uma via lateral e outra via na região medial $^{7}$. Atenção especial deve ser dada aos sinais neurológicos. Pesquisas apontam que cerca de $12 \%$ a $24 \%$ dos casos tratados surgem comprometimentos neurológicos, desses de $2 \%$ a $6 \%$ correspondem a lesão neurológica iatrogênica. Não é tão comum encontrar a lesão vascular, mas, mesmo assim, pode estar presente em aproximadamente $10 \%$ dessas fraturas, pelo que se torna imperativo a identificação precoce de sinais de compromisso vascular para um tratamento adequado a fim de prevenir futuras sequelas ${ }^{8}$.

O presente trabalho objetiva explanar a fratura exposta supracondiliana umeral com lesão vásculo-nervosa em paciente pediátrico por meio de relato de caso e estudos de embasamento teórico.

\section{CASO CLÍNICO}

Paciente do sexo masculino, sete anos de idade, atendido em emergência após queda de cavalo e traumatismo em cotovelo esquerdo com exposição óssea em ferimento maior do que dez centímetros. Reconhecida como fratura exposta supracondiliana em cotovelo esquerdo - grau 3C - com lesão da artéria braquial e dos nervos radial e ulnar (Figuras 1 e 2). Pulsos radial e ulnar ausentes, palidez cutânea e déficit do nervo radial e ulnar. $O$ paciente foi encaminhado imediatamente para centro cirúrgico, onde foi realizada lavagem exaustiva com oito litros de soro fisiológico 0,9\%, desbridamento cirúrgico, redução e fixação da fratura com três fios de Kirschner número 2. Foi realizada revascularização da artéria braquial e neurorrafia dos nervos radial e ulnar. Fechamento por planos, curativo e tala axilopalmar com antibioticoterapia (Figura 3).

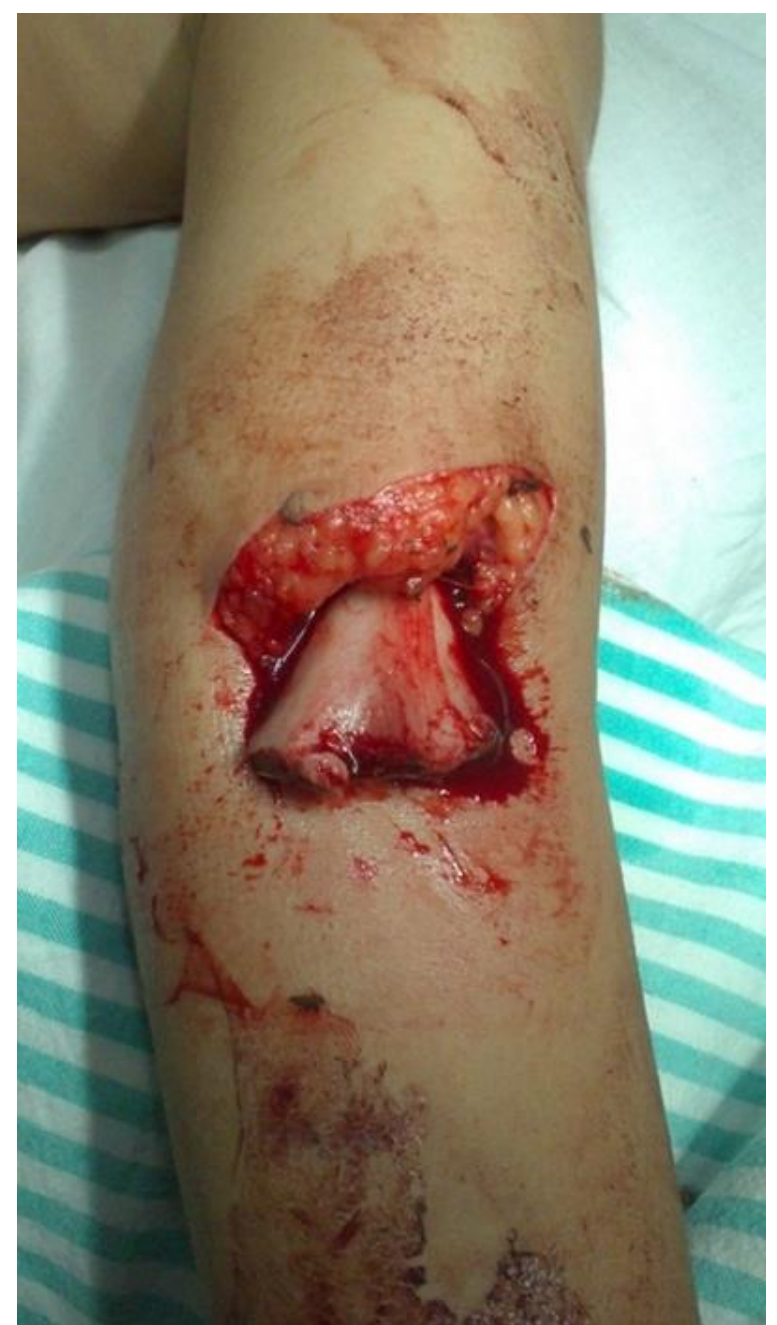

Figura 1: Fratura exposta supracondiliana Grau 3C.

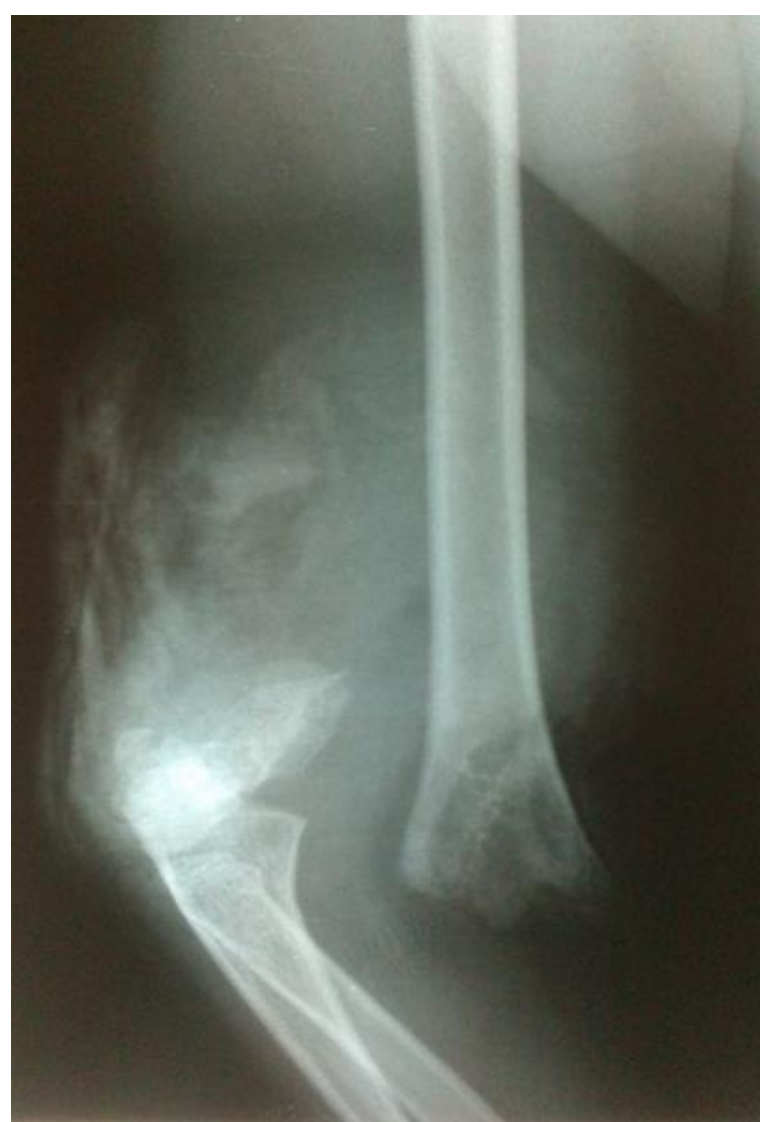

Figura 2: Radiografia realizada como exame complementar. 
Ocorreu evolução com retorno da perfusão periférica e pulsos radial e ulnar. Foi encaminhado para o ambulatório de ortopedia. Transcorridos três semanas, houve consolidação da fratura com pulsos radial e ulnar preservados, mas com déficits nos nervos radial e ulnar.

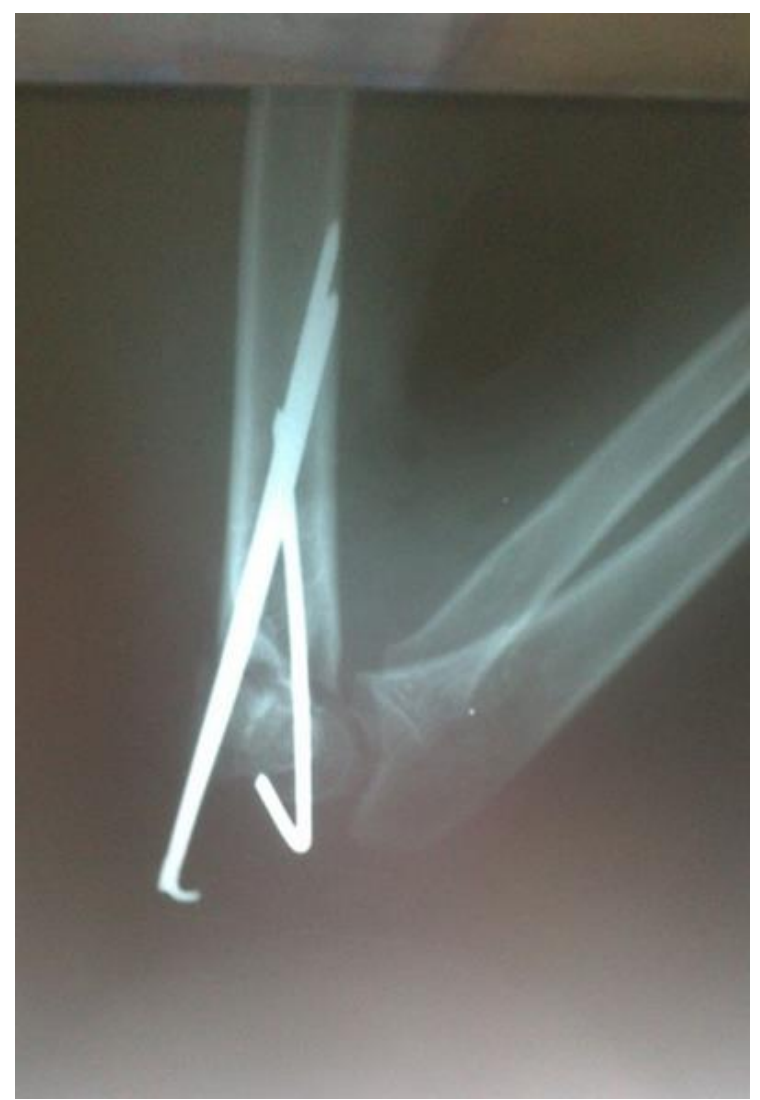

Figura 3. Radiografia realizada após o procedimento cirúrgico.

Retirados os três fios, foi encaminhado para reabilitação. Transcorridos dois meses de reabilitação, já apresentava arco total do movimento do cotovelo, porém mantinha déficits radial e ulnar. Com seis meses de reabilitação, mostrava esboço dos nervos radial e ulnar e com um ano retorno de suas funções normais.

DISCUSSÃO

Ainda existem contradições em relação a melhor escolha do caminho a ser seguido no tratamento, justamente por ser uma região delicada e repleta de situações anatômicas que não dizem respeito apenas a ossificação da fratura, mas sim, a um complexo sistema integrado por ossos, ligamentos, músculos, sistema vascular e neurológico que propiciam muitas vezes o processo iatrogênico durante a intervenção cirúrgica ${ }^{9}$.

As fraturas por extensão são as mais frequentes. Nas fraturas supracondilianas do úmero as lesões vasculares (artéria umeral/braquial), as lesões nervosas, do nervo mediano, do nervo cubital e do nervo radial e a exposição cutânea dos topos ósseos são as mais evidenciadas. Nesse sentido, o exame vasculonervoso do membro superior tem relativizada sua elevada importância. Se a dúvida persistir outra indicação poderá ser o estudo da artéria braquial com eco-doppler ou eventualmente uma arteriografia, já que podem existir feridas arteriais e dissecções subadventícias traumáticas, que por si só justificam a urgência de reconstrução vascular. Assim, pode-se evitar graves sequelas, desde uma amputação por isquemia até uma contratura isquêmica de Volkman ${ }^{6,10}$.

Não há consenso, tanto na literatura quanto nos estudos comparativos, sobre o tipo de fixação que deve ser utilizada, a cruzada ou lateral. O mesmo problema é enfrentado no que diz respeito a indicação para tração esquelética. Ambos os procedimentos dependem muito da conduta de cada cirurgião e dos tipos de fratura desse estilo, já que podem ser classificadas e deter diferentes abordagens e manejos ${ }^{11}$.

Também não há consenso no uso da fisioterapia para reabilitação pós-cirúrgica e eventualmente, na literatura, não há boas recomendações, ainda mais se os resultados forem a longo prazo (cerca de um ano de diferença) quando comparados, isso se o paciente não possuir déficit muscular ${ }^{12}$.

As complicações mais comuns de acontecer são: diminuição do arco do movimento de extensão, perda do movimento de flexão, deformidade angular (cúbito varo), transtorno do crescimento da coluna medial, capítulo ou fise da tróclea, cúbito valgo e paralisia do nervo ulnar. A maioria destas acontecem devido a erro da instituição e do processo terapêutico ${ }^{12}$.

Um dos fatores que causa bastante divergência é a questão da abordagem desse tipo de lesão quanto a identificar e decidir a conduta que deve ser tomada na falta de pulso e perfusão. Salienta-se que a ausência de pulso não significa lesão do sistema vascular, bem como sua presença não a descarta, mas devem ser identificados e valorizados, visto servirem para avaliação da evolução do paciente, sobretudo nas primeiras doze horas desde a ocorrência do trauma ${ }^{10,12.14}$.

A existência de fratura com deformidade póstero-lateral significativa deve alertar para a existência de lesão muscular grave e isquemia da musculatura adjacente ao foco de fratura, a presença de dor em repouso, dor provocada pela extensão passiva dos dedos e a incapacidade de contrair ativamente os músculos do antebraço devem alertar para a possibilidade de transtorno vascular da região ${ }^{10,12,13}$. 


\section{CONFLITO DE INTERESSES}

Os autores declaram não haver conflitos de interesse

\section{AUTOR PARA CORRESPONDÊNCIA}

\section{Amanda Oliva Spaziani}

E-mail: spazianimedicina@gmail.com

Submetido em 27/10/2019

Aceito em 31/08/2020 atura-supracondiliana-do-umero-na-crianca.pdf

3. Placzek JD, Boyce, DA. Segredos da fisioterapia ortopédica. Porto Alegre: Artmed; 2001.

4. Schwartsmann C, Lech O, Telöken M. Fraturas: princípios e prática. Porto Alegre: Artmed; 2003.

5. Gartland JJ. Management of supracondylar fractures of the humerus in children. Surg Gynecol Obstet. 1959;109(2):145-54.

6. Judas $F$, Matos $P$. Patologia cirúrgica do cotovelo, antebraço, punho e mão: conceitos essenciais [dissertação]. Coimbra: Universidade de Coimbra; 2011.

7. Zoppi Filho A, Kikuta FK, Mouraria GG, Zan RA. Via de acesso global ao cotovelo. Rev Ortop llustrada. 2012;3(1):14-9.

8. Henrikson B. Supracondylar fracture of the humerus in children. A late review of endresults with special reference to the cause of deformity, disability and complications. Acta Chir Scand Suppl. 1966;369:1-72.

9. Gandhi J, Horne G. Fraturas supracondilares tipo III do úmero em crianças: tratamento com braço reto. Acta ortop bras. 2010;18(3):132-34.

10. Patriota GSQA, Assunção Filho CA, Assunção CA. Qual a melhor técnica para fixação no tratamento de fratura supracondilar do úmero em crianças? Rev Bras Ortop [Internet]. 2017;52(4):428-34.

11. Moreira RFG, Nishimi AY, Zanon EM, Rama TS, Ciofi RPL, Dobashi ET. Estudo transversal sobre 0 tratamento das fraturas supracondilianas do úmero na infância dos tipos Gartland II e III: opinião do ortopedista brasileiro. Rev Bras Ortop. 2018;53(2):129-35.

12. Rocha ITS, Faria AS, Fontoura Filho C, Rocha MA. Reprodutibilidade das classificações AO/ASIF e Gartland para fraturas supracondilianas de úmero em crianças. Rev Bras Ortop. 2015;50(3):266-69.

13. Mubarak SJ, Carroll NC. Volkmann's contracture in children: aetiology and prevention. J Bone Joint Surg Br. 1979;61$B(3): 285-93$. 